\title{
FINSLER GEODESICS, PERIODIC REEB ORBITS, AND OPEN BOOKS
}

\author{
MAX DÖRNER, HANSJÖRG GEIGES, AND KAI ZEHMISCH
}

\begin{abstract}
We survey some results on the existence (and non-existence) of periodic Reeb orbits on contact manifolds, both in the open and closed case. We place these statements in the context of Finsler geometry by including a proof of the folklore theorem that the Finsler geodesic flow can be interpreted as a Reeb flow. As a mild extension of previous results we present existence statements on periodic Reeb orbits on contact manifolds with suitable supporting open books.
\end{abstract}

\section{INTRODUCTION}

From a Riemannian perspective, Reeb flows on contact manifolds may be regarded as a generalisation of the geodesic flow on a Finsler manifold. It is therefore reasonable to ask to what extent statements about geodesic flows are instances of more general facts in Reeb dynamics.

In this brief survey, we are interested in existence and non-existence statements about periodic Reeb orbits. Recall that a contact form on a $(2 n+1)$-dimensional manifold is a 1-form $\alpha$ such that $\alpha \wedge(\mathrm{d} \alpha)^{n}$ is nowhere zero, i.e. a volume form. The Reeb vector field $R_{\alpha}$ of $\alpha$ is uniquely defined by the equations

$$
\left.R_{\alpha}\right\lrcorner \mathrm{d} \alpha=0 \text { and } \alpha\left(R_{\alpha}\right)=1,
$$

where $\lrcorner$ denotes the interior product.

A (cooriented) contact structure $\xi \subset T M$ is the maximally non-integrable tangent hyperplane field defined as the kernel of a contact form $\alpha$; this contact form is unique up to multiplication with a positive function. A contact manifold $(M, \xi)$ is said to satisfy the Weinstein conjecture [47, if

(W) the Reeb vector field of every contact form defining $\xi$ has a periodic Reeb orbit.

For closed manifolds, this conjecture has been established under various assumptions on the topology of $M$ or particular properties of $\xi$ (such as so-called overtwistedness). In its full generality, the conjecture remains open. There are simple examples of open manifolds where (W) fails, but recently there has been some progress on establishing $(\mathrm{W})$ for a number of open contact manifolds.

A stronger requirement is that

$\left(\mathrm{W}^{\circ}\right)$ the Reeb vector field of every contact form defining $\xi$ has a contractible periodic orbit.

2010 Mathematics Subject Classification. 37J45; 37C27, 53B40, 53D25, 53D35.

The research reported in this survey is supported by the Deutsche Forschungsgemeinschaft (GE 1245/2-1 to H.G. and ZE 992/1-1 to K.Z.) and is part of a project in the SFB/TRR 191 'Symplectic Structures in Geometry, Algebra and Dynamics'. 
Here the period for which the orbit is contractible is not required to be the minimal one. Even with this relaxation, one cannot expect $\left(\mathrm{W}^{\circ}\right)$ to hold for all closed contact manifolds. Take, for instance, the 3-torus $S^{1} \times S^{1} \times S^{1}$ with the contact form $\sin \theta \mathrm{d} x-\cos \theta \mathrm{d} y$. The Reeb vector field $\sin \theta \partial_{x}-\cos \theta \partial_{y}$ has plenty of periodic Reeb orbits, all of them non-contractible.

The fact that the Finsler geodesic flow is a Reeb flow has been mentioned in various sources, but we have not been able to find a reference that includes a complete proof. Therefore, as a service to the reader, we present such a demonstration in Section 2. In Section 3] we argue that it is expedient to view Finsler geodesic flows on the cotangent rather than the tangent bundle, where Reeb flows appear not as an ad hoc interpretation, but as the natural generalisation of Finsler geodesic flows.

In Section 4 we discuss the existence and non-existence of periodic Reeb orbits on non-compact manifolds. The last two sections of this survey deal with the Weinstein conjecture on closed manifolds. It is shown how information on the topology of the manifold allows one to prove $(\mathrm{W})$ or $\left(\mathrm{W}^{\circ}\right)$. In Section [5, the information on the topology comes from surgery; in Section 6, from open book decompositions. This last section contains instances of $(\mathrm{W})$ or $\left(\mathrm{W}^{\circ}\right)$ that have not previously appeared in the literature.

\section{Finsler geodesics as Reeb orbits}

It is a folklore result that the Finsler geodesic flow can be viewed as a Reeb flow, see [7, Theorem 1.1.2] or [30], for instance. Here we present a complete proof of this fact, inspired by the remark in [7, p. 6] that one should identify the defining equations for the Reeb vector field as the Euler-Lagrange equations for the length functional.

On a manifold $Q$, we write tangent vectors as $(\mathbf{q}, \mathbf{v}) \in T_{\mathbf{q}} Q$ or simply $\mathbf{v}$ if the base point is clear from the context. Local coordinates on the tangent bundle $T Q$ are chosen such that

$$
\left(q^{1}, \ldots, q^{n}, v^{1}, \ldots, v^{n}\right)=\left(v^{i} \frac{\partial}{\partial q^{i}}\right)_{\left(q^{1}, \ldots, q^{n}\right)},
$$

with summation convention understood. A Finsler metric on $Q$ is a function $F: T Q \rightarrow \mathbb{R}_{0}^{+}$on the tangent bundle with the following properties:

(i) $G:=F^{2} / 2$ is smooth outside the zero section $Q \subset T Q$.

(ii) $F(\mathbf{q}, \mathbf{v})=0$ if and only if $\mathbf{v}=\mathbf{0}$.

(iii) $F(\mathbf{q}, \rho \mathbf{v})=\rho F(\mathbf{q}, \mathbf{v})$ for $\rho \in \mathbb{R}^{+}$.

(iv) $G$ is strictly convex, i.e. at each point $\mathbf{q} \in Q$ the function $\mathbf{v} \mapsto G(\mathbf{q}, \mathbf{v})$ on $T_{\mathbf{q}} Q \backslash\{\mathbf{0}\}$ has a positive definite Hessian matrix.

Condition (iv) implies that the sublevel sets of $G$ (or $F$ ) are strictly convex, cf. [18, Lemma 5.3.1].

A Riemannian metric $g$ on $Q$ gives rise to a Finsler metric by setting $F(\mathbf{q}, \mathbf{v})=$ $\left(g_{\mathbf{q}}(\mathbf{v}, \mathbf{v})\right)^{1 / 2}$. In this case $G$ is smooth on all of $T Q$. In fact, this smoothness property at the zero section characterises Finsler metrics coming from a Riemannian metric, see [1, Lemma 1.4.1].

The length of a smooth curve $\gamma:[a, b] \rightarrow Q$ with respect to the Finsler metric $F$ is defined as

$$
L(\gamma)=\int_{a}^{b} F(\gamma(t), \dot{\gamma}(t)) \mathrm{d} t
$$


The critical points of this length functional are the Finsler geodesics when parametrised proportional to arc length. For a characterisation of Finsler geodesics as critical points of an energy functional see [39, 40]; here the parametrisation proportional to arc length is automatic.

We do not require the Finsler metric to be reversible, i.e. we do not impose the condition $F(\mathbf{q},-\mathbf{v})=F(\mathbf{q}, \mathbf{v})$. Consequently, the orientation of a curve is essential for its being a geodesic. Nonetheless, as in the Riemannian setting, geodesics are defined by a geodesic vector field on the tangent bundle, defining a directed geodesic flow. Thus, through every point in $T Q \backslash Q$ there is a unique directed curve projecting to a geodesic; see [1, Section 1.5].

In order to relate Finsler geodesics to Reeb flows, we rewrite this length functional in terms of the so-called Hilbert form. In local coordinates on $T Q \backslash Q$, this 1 -form is defined as

$$
\beta:=\frac{\partial F}{\partial v^{i}} \mathrm{~d} q^{i}
$$

Given different local coordinates $\left(x^{j}, w^{j}\right)$, we have

$$
v^{i}=\frac{\partial q^{i}}{\partial x^{j}} w^{j}
$$

whence

This implies

$$
\frac{\partial v^{i}}{\partial w^{j}}=\frac{\partial q^{i}}{\partial x^{j}}
$$

$$
\frac{\partial F}{\partial w^{j}} \mathrm{~d} x^{j}=\frac{\partial F}{\partial v^{i}} \frac{\partial v^{i}}{\partial w^{j}} \mathrm{~d} x^{j}=\frac{\partial F}{\partial v^{i}} \frac{\partial q^{i}}{\partial x^{j}} \mathrm{~d} x^{j}=\frac{\partial F}{\partial v^{i}} \mathrm{~d} q^{i},
$$

which shows that the Hilbert form $\beta$ is globally defined on $T Q \backslash Q$.

Observe that by Euler's theorem on homogeneous functions and the homogeneity condition (iii) we have $v^{i}\left(\partial F / \partial v^{i}\right)=F$. This allows one to rewrite the arc length integral as an integral of the Hilbert form, where $\Gamma=(\gamma, \dot{\gamma})$ is the curve on $T Q \backslash Q$ defined by a regular curve $\gamma$ on $Q$ :

$$
L(\gamma)=\int_{a}^{b} F(\gamma(t), \dot{\gamma}(t)) \mathrm{d} t=\int_{a}^{b} \frac{\partial F}{\partial v^{i}}(\gamma(t), \dot{\gamma}(t)) \dot{\gamma}^{i}(t) \mathrm{d} t=\int_{\Gamma} \beta .
$$

It is well known that the Hilbert form $\beta$ is a contact form on the level sets of $F$ (or on the projectivised tangent bundle), see [11, Chapter 8]. However, for the relation with symplectic geometry, it seems to be more convenient to work with the 1 -form

$$
\alpha:=F \beta=\frac{\partial G}{\partial v^{i}} \mathrm{~d} q^{i} .
$$

We are going to show that $\alpha$ is a contact form on each level set of $F$, and hence so is $\beta$.

Proposition 2.1. The 2 -form $\mathrm{d} \alpha$ is a symplectic form on $T Q \backslash Q$.

Proof. We compute

$$
\mathrm{d} \alpha=\frac{\partial^{2} G}{\partial v^{i} \partial v^{j}} \mathrm{~d} v^{j} \wedge \mathrm{d} q^{i}+\frac{\partial^{2} G}{\partial v^{i} \partial q^{j}} \mathrm{~d} q^{j} \wedge \mathrm{d} q^{i} .
$$

For the $n$-fold wedge product $(\mathrm{d} \alpha)^{n}$, only the first summand is relevant, and $\mathrm{d} \alpha$ being symplectic is seen to be a consequence of the convexity condition (iv). 
A computation as above shows that the fibrewise radial vector field $V:=v^{i} \frac{\partial}{\partial v^{i}}$ is independent of the choice of local coordinates. This also follows from the next lemma.

Lemma 2.2. The radial vector field $V$ satisfies $V\lrcorner \mathrm{d} \alpha=\alpha$ and $V\lrcorner \mathrm{d} \beta=0$.

Proof. Euler's theorem on homogeneous functions gives us

$$
v^{k} \frac{\partial G}{\partial v^{k}}=2 G
$$

Differentiating this equation with respect to $v^{i}$, we find

$$
v^{k} \frac{\partial^{2} G}{\partial v^{i} \partial v^{k}}=\frac{\partial G}{\partial v^{i}}
$$

Now we compute

$$
\begin{aligned}
V\lrcorner \mathrm{d} \alpha & \left.=v^{k} \frac{\partial}{\partial v^{k}}\right\lrcorner \frac{\partial^{2} G}{\partial v^{i} \partial v^{j}} \mathrm{~d} v^{j} \wedge \mathrm{d} q^{i} \\
& =v^{k} \frac{\partial^{2} G}{\partial v^{i} \partial v^{k}} \mathrm{~d} q^{i} \\
& =\frac{\partial G}{\partial v^{i}} \mathrm{~d} q^{i}=\alpha .
\end{aligned}
$$

Based on the corresponding identity

$$
v^{k} \frac{\partial^{2} F}{\partial v^{i} \partial v^{k}}=0
$$

for $F$, the computation showing that $V \downarrow \mathrm{d} \beta=0$ is similar.

With the Cartan formula for the Lie derivative it follows that $V$ is a Liouville vector field with respect to the symplectic form $\mathrm{d} \alpha$, that is, $L_{V} \mathrm{~d} \alpha=\mathrm{d} \alpha$. Moreover, the homogeneity condition (iii) guarantees (by Euler's theorem) that $V$ is transverse to the level sets of $F$. This entails that $\alpha$ induces a contact form on each level set, cf. [18, Lemma 1.4.5].

Write $M$ for the level set $F^{-1}(1)$. On this level set we have the contact form $\left.\alpha\right|_{T M}=\left.\beta\right|_{T M}$ with Reeb vector field $R$. Since $V \downarrow \mathrm{d} \beta=0$, the kernel of the 2 -form $\mathrm{d} \beta \mid M$ is spanned by $R$ and $V$. Also, we have $\beta(V)=0$. Hence, the Reeb vector field $R$ of $\left.\beta\right|_{T M}$ can equivalently be defined as follows. Let $\widetilde{R}$ be a section of $T(T Q)$ along $M$ such that

$$
\widetilde{R}\lrcorner \mathrm{d} \beta=0 \text { on }\left.T(T Q)\right|_{M} \text { and } \beta(\widetilde{R})=1 .
$$

Then $R$ equals the projection of $\widetilde{R}$ onto $T M$ along the line bundle $\langle V\rangle$.

Lemma 2.3. A local solution $\widetilde{R}$ of (2) is given by

$$
\widetilde{R}=v^{i} \frac{\partial}{\partial q^{i}}+a^{i} \frac{\partial}{\partial v^{i}}
$$

where $\left(a^{1}, \ldots, a^{n}\right)$ is a solution of the equations

$$
v^{k} \frac{\partial^{2} F}{\partial v^{i} \partial q^{k}}-v^{k} \frac{\partial^{2} F}{\partial v^{k} \partial q^{i}}+a^{k} \frac{\partial^{2} F}{\partial v^{i} \partial v^{k}}=0, \quad i=1, \ldots, n .
$$


Proof. We first convince ourselves that (3) has a solution. Along $M=F^{-1}(1)$ we have

$$
\frac{\partial^{2} G}{\partial v^{i} \partial v^{k}}=\frac{\partial^{2} F}{\partial v^{i} \partial v^{k}}+\frac{\partial F}{\partial v^{i}} \frac{\partial F}{\partial v^{k}} .
$$

Write $F_{i}=\partial F / \partial v^{i}$ for brevity, similar for higher derivatives. The matrix

$$
\left(F_{i} F_{k}\right)=\left(F_{1}, \ldots, F_{n}\right)^{\mathrm{t}}\left(F_{1}, \ldots, F_{n}\right)
$$

has rank 1 . The matrix $\left(G_{i k}\right)$ has rank $n$ by the convexity condition (iv). It follows that $\left(F_{i k}\right)$ has rank at least equal to $n-1$. Since the vector $\left(v^{1}, \ldots, v^{n}\right)$ lies in the kernel of this last matrix, its rank equals $n-1$, and the image of this symmetric matrix is the subspace of $\mathbb{R}^{n}$ orthogonal to $\left(v^{1}, \ldots, v^{n}\right)$. It follows that (3) indeed has a solution $\left(a^{1}, \ldots, a^{n}\right)$, unique up to adding multiples of $\left(v^{1}, \ldots, v^{n}\right)$.

Given such a solution, let $\widetilde{R}$ be as defined in the lemma. We first check

$$
\beta(\widetilde{R})=v^{i} \frac{\partial F}{\partial v^{i}}=F=1 \text { on } M
$$

With the help of (11) and (31) one easily verifies the first condition in (2).

Consequently, the Reeb vector field $R$ is of the form

$$
R=v^{i} \frac{\partial}{\partial q^{i}}+b^{i} \frac{\partial}{\partial v^{i}}
$$

This means that Reeb orbits in $M \subset T Q$ are of the form $(\gamma, \dot{\gamma})$, where $\gamma$ is a unit speed trajectory in $Q$.

Proposition 2.4. The unit speed geodesics on $Q$ with respect to the Finsler metric $F$ are precisely the projections of Reeb orbits on $M$.

Proof. Let $\gamma:[a, b] \rightarrow Q$ be a unit speed curve, and write $\Gamma=(\gamma, \dot{\gamma})$ for the corresponding curve in $M$. Consider a variation $\Gamma_{s}=\left(\gamma_{s}, \dot{\gamma}_{s}\right), s \in(-\varepsilon, \varepsilon)$, of curves in $T Q \backslash Q$, fixed near the endpoints. Write

$$
X=\left.\frac{\mathrm{d} \Gamma_{s}}{\mathrm{~d} s}\right|_{s=0}
$$

for the variational vector field at $s=0$. Then

$$
\left.\left.\frac{\mathrm{d}}{\mathrm{d} s}\right|_{s=0} L\left(\gamma_{s}\right)=\left.\frac{\mathrm{d}}{\mathrm{d} s}\right|_{s=0} \int_{a}^{b} \Gamma_{s}^{*} \beta=\int_{\Gamma}(\mathrm{d}(\beta(X))+X\lrcorner \mathrm{d} \beta\right) ;
$$

see [18, Lemma B.1] for the last equality. The integral over the first summand vanishes since the variation is fixed near the end points. So the condition for $\gamma$ to be a geodesic, that is, the vanishing of this first variation, becomes

$$
\int_{a}^{b} \mathrm{~d} \beta(X, \dot{\Gamma}) \mathrm{d} t=0 .
$$

It would be precipitate to conclude that this forces $\dot{\Gamma}$ to equal (a multiple of) the Reeb vector field, since only a subclass of vector fields arises as variational vector fields $X$. However, for Reeb trajectories this first variation certainly vanishes, even under general variations. Moreover, we have seen that Reeb trajectories are of the form $(\gamma, \dot{\gamma})$, with $\gamma$ of unit speed. This implies that every Reeb orbit does indeed project to a Finsler geodesic.

Conversely, through every point $(\mathbf{q}, \mathbf{v}) \in M \subset T Q$ there is a unique curve projecting to a unit speed geodesic, so this curve coincides with the Reeb orbit through that point. 
Remark 2.5. For a particular case of this result, the geodesic flow of a Riemannian manifold, a proof can be found in [18, Section 1.5]. The proof above is simpler, since it avoids computations in special local coordinates.

A proof of the general case, using a different line of reasoning and written in Portuguese, can be found in 31, where, in Teorema 4.4.10, the Hilbert form is identified with the pull-back of the canonical Liouville 1-form on the unit cotangent bundle, and the geodesic spray with the Reeb vector field.

\section{The DUAL VIEWPOINT}

In this section we expand a little on that last remark and explain why Finsler geodesic flows have a more natural interpretation in the cotangent bundle. We thank Felix Schlenk [42] and the referees for useful suggestions regarding this issue.

On the cotangent bundle $T^{*} Q$ we choose local coordinates such that

$$
\left(q^{1}, \ldots, q^{n}, p_{1}, \ldots, p_{n}\right)=\left(p_{i} \mathrm{~d} q^{i}\right)_{\left(q^{1}, \ldots, q^{n}\right.} \cdot
$$

In these coordinates, and the adapted coordinates on $T Q$ as in the preceding section, the Legendre transformation $\mathcal{L}: T Q \backslash Q \rightarrow T^{*} Q \backslash Q$ defined by the Lagrange function $G: T Q \rightarrow \mathbb{R}$ is described by

$$
\left(q^{1}, \ldots, q^{n}, v^{1}, \ldots, v^{n}\right) \longmapsto\left(q^{1}, \ldots, q^{n}, p_{1}=\frac{\partial G}{\partial v^{1}}, \ldots, p_{n}=\frac{\partial G}{\partial v^{n}}\right),
$$

see [35, 8.3]. The homogeneity and strong convexity of $G$ ensure that $\mathcal{L}$ is a diffeomorphism. Under this diffeomorphism, the canonical Liouville form $\lambda_{\text {can }}=p_{i} \mathrm{~d} q^{i}$ on $T^{*} Q$ pulls back to the contact form on $T Q$ that we called $\alpha$.

Remark 3.1. In the Riemannian case, when $G=g_{i j} v^{i} v^{j} / 2$, we have $\partial G / \partial v^{i}=$ $g_{i j} v^{j}$, so the Legendre transformation coincides with the identification of $T Q$ with $T^{*} Q$ given by the metric. For this case see also [18, Theorem 1.5.2].

The strict convexity of the unit ball $B_{\mathbf{q}} \subset T_{\mathbf{q}} Q$ with respect to $F$,

$$
B_{\mathbf{q}}:=\left\{\mathbf{v} \in T_{\mathbf{q}} Q: G(\mathbf{q}, \mathbf{v}) \leq 1 / 2\right\},
$$

implies that for $\mathbf{v} \in \partial B_{\mathbf{q}}$ and $\mathbf{w}$ a tangent vector to the fibre $T_{\mathbf{q}} Q$ at $\mathbf{v} \in T_{\mathbf{q}} Q$ we have

$$
\frac{\partial G}{\partial v^{i}} w^{i} \leq \frac{\partial G}{\partial v^{i}} v^{i}=2 G=1,
$$

with equality only for $\mathbf{w}=\mathbf{v}$ (interpreting $\mathbf{w}$ as an element of $T_{\mathbf{q}} Q$ ). So the polar body $B_{\mathbf{q}}^{*}:=\mathcal{L}\left(B_{\mathbf{q}}\right) \subset T_{\mathbf{q}}^{*} Q$ is the set

$$
B_{\mathbf{q}}^{*}=\left\{\mathbf{p} \in T_{\mathbf{q}}^{*} Q: p_{i} v^{i} \leq 1 \text { for all } \mathbf{v} \in B_{\mathbf{q}}\right\} .
$$

Since $G$ is fibrewise homogeneous of degree 2 , the Hamiltonian function $G^{*}: T^{*} Q \rightarrow$ $\mathbb{R}$ corresponding to $G$ is simply given by $G^{*}=G \circ \mathcal{L}^{-1}$, see [35, 8.6]. In other words, $G^{*}$ takes the value $1 / 2$ on the boundary of $B_{\mathbf{q}}^{*}$ and is likewise fibrewise homogeneous of degree 2 .

It now becomes perfectly obvious why the Finsler geodesic flow is a Reeb flow. The Finsler geodesics are the solutions of the Lagrangian system defined by the function $G$ on $T Q$, and they transform under $\mathcal{L}$ to the solutions of the Hamiltonian system on $T^{*} Q$ (equipped with the canonical symplectic form $\omega_{\text {can }}=\mathrm{d} p_{i} \wedge \mathrm{d} q^{i}$ ) defined by $G^{*}$, see [35, Chapter 10]. For the following lemma cf. [16, Lemma 4.2] and [18, Lemma 1.4.10]. 
Lemma 3.2. The Hamiltonian flow of $G^{*}$ on the unit cotangent bundle $M:=$ $S T^{*} Q=\left\{G^{*}=1 / 2\right\}$ coincides with the Reeb flow of the contact form $\left.\lambda_{\text {can }}\right|_{T M}$ given by the restriction of the Liouville form.

Proof. Both the Hamiltonian vector field $\left.X_{G^{*}}\right|_{M}$ and the Reeb vector field of $\left.\lambda_{\text {can }}\right|_{T M}$ span the kernel of $\left.\omega_{\text {can }}\right|_{T M}$. Write $Y=p_{i} \frac{\partial}{\partial p_{i}}$ for the fibrewise radial vector field on $T^{*} Q$, so that $\left.\lambda_{\text {can }}=Y\right\lrcorner \omega_{\text {can }}$. From the computation

$$
\lambda_{\text {can }}\left(X_{G^{*}}\right)=\omega_{\text {can }}\left(Y, X_{G^{*}}\right)=\mathrm{d} G^{*}(Y)=2 G^{*}=1 \text { on } M,
$$

where we have used the homogeneity of $G^{*}$, we conclude that $\left.X_{G^{*}}\right|_{M}$ coincides with the Reeb vector field.

Rescaling the contact form $\left.\lambda_{\text {can }}\right|_{T M}$ by a positive function is equivalent to taking the induced contact form on a fibrewise starshaped hypersurface in $T^{*} Q$. In this way, a large class of Reeb flows arises as a natural generalisation of the Finsler cogeodesic flow.

Finsler (co-)metrics come from fibrewise strictly convex hypersurfaces enclosing the zero section in $T Q$ and $T^{*} Q$, respectively. Both viewpoints are equivalent under the Legendre transformation. In the cotangent bundle, there is a natural extension of Finsler cogeodesic flows to the rich theory of Reeb flows on fibrewise starshaped hypersurfaces enclosing the zero section, see [16. Here the Legendre transformation breaks down, so there is no correspondence with metrics on $Q$ defined by fibrewise starshaped hypersurfaces in the tangent bundle.

Indeed, as pointed out by Busemann [10, p. 83], if one drops the condition that $\{F=1\} \cap T_{\mathbf{q}} Q$ be convex in $T_{\mathbf{q}} Q$ for all $\mathbf{q} \in Q$, the resulting metric geometry has some awkward features. Given any function $F: T Q \rightarrow \mathbb{R}_{0}^{+}$satisfying conditions (i) to (iii) as in the preceding section, one can define the $F$-length $L(\gamma)=\int F(\gamma, \dot{\gamma})$ of a (piecewise) $C^{1}$-curve $\gamma$ in $Q$. The distance between two points $\mathbf{q}_{0}, \mathbf{q}_{1}$ in $Q$ is naturally set to be the infimum over the $F$-length of all $C^{1}$-curves joining $\mathbf{q}_{0}$ with $\mathbf{q}_{1}$. With the help of this distance function one can then talk about rectifiable curves and their arc length obtained by polygonal approximation. However, this arc length will coincide with the $F$-length on all $C^{1}$-curves only if the unit spheres in $T_{\mathbf{q}} Q$ with respect to $F$ are convex for all $\mathbf{q} \in Q$.

To summarise, only when viewing Finsler geometry on the cotangent rather than the tangent bundle do we perceive a natural and fruitful generalisation of Finsler geodesic flows, first to Reeb flows on fibrewise starshaped hypersurfaces, thence to general contact manifolds.

\section{EXISTENCE VS. NONEXISTENCE}

On non-compact contact manifolds, the Reeb vector field will not, in general, have any periodic orbits. Write

$$
\alpha_{\mathrm{st}}=\mathrm{d} z+\frac{1}{2} \sum_{j=1}^{n}\left(x_{j} \mathrm{~d} y_{j}-y_{j} \mathrm{~d} x_{j}\right)
$$

for the standard contact form on $\mathbb{R}^{2 n+1}$, and $\xi_{\text {st }}=\operatorname{ker} \alpha_{\text {st }}$ for the standard contact structure. The Reeb vector field of $\alpha_{\mathrm{st}}$ is the coordinate vector field $\partial_{z}$. 
We also write $\xi_{\text {st }}=\operatorname{ker} \alpha_{\text {st }}$ for the standard contact structure and contact form, respectively, on the sphere $S^{2 n+1} \subset \mathbb{R}^{2 n+2}$, defined by

$$
\alpha_{\text {st }}=\sum_{j=1}^{n+1}\left(x_{j} \mathrm{~d} y_{j}-y_{j} \mathrm{~d} x_{j}\right) .
$$

This notational duplication is justified by the fact that the two contact structures $\xi_{\text {st }}$ on $\mathbb{R}^{2 n+1}$ and on the complement of a point in $S^{2 n+1}$, respectively, are diffeomorphic, see [18, Proposition 2.1.8]. The Reeb vector field of $\alpha_{\text {st }}$ on $S^{2 n+1}$ is given by

$$
\sum_{j=1}^{n+1}\left(x_{j} \partial_{y_{j}}-y_{j} \partial_{x_{j}}\right),
$$

so all Reeb orbits are periodic, and they define the generalised Hopf fibration $S^{1} \hookrightarrow$ $S^{2 n+1} \rightarrow \mathbb{C P}^{n}$.

Going back to $\left(\mathbb{R}^{2 n+1}, \alpha_{\text {st }}\right)$, it is natural to ask whether local changes in the topology or the contact form force the existence of periodic Reeb orbits. One very simple way to make a local change in the contact form, but no change in the topology, that will produce a periodic Reeb orbit is to take a contact connected sum with $\left(S^{2 n+1}, \alpha_{\text {st }}\right)$ inside a small ball in $\mathbb{R}^{2 n+1}$; see also the next section. For the definition of contact surgery we refer the reader to [48, and [18, Chapter 6].

An essential tool for finding or excluding periodic Reeb orbits are holomorphic discs. To start with the most simple example, consider the cylinder

$$
Z=D^{2} \times \mathbb{R}=\left\{x^{2}+y^{2} \leq 1\right\} \subset \mathbb{R}^{3} .
$$

Let $\alpha$ be a contact form on $\mathbb{R}^{3}$ that coincides with $\alpha_{\text {st }}$ outside a small (not necessarily round) ball $B$ contained in $Z$. Equip the symplectisation $\left(\mathbb{R} \times \mathbb{R}^{3}, \omega:=\mathrm{d}\left(\mathrm{e}^{t} \alpha\right)\right)$ with an almost complex structure $J$ which is tamed by $\omega$, that is, $\omega(X, J Y)$ defines a $J$-invariant Riemannian metric, and which satisfies $J\left(\partial_{t}\right)=R_{\alpha}$. Away from $\mathbb{R} \times B$ we take $J$ to be the standard complex structure $J\left(\partial_{x}\right)=\partial_{y}, J\left(\partial_{t}\right)=\partial_{z}$. Then the domain $(-\infty, 0] \times Z$ has a piecewise smooth strictly pseudoconvex boundary.

One now studies holomorphic discs

$$
(a, f): \mathbb{D}^{2} \longrightarrow \mathbb{R} \times Z
$$

whose boundary map $\partial \mathbb{D}^{2} \rightarrow\{0\} \times \partial Z$ is of degree one. Away from $\mathbb{R} \times B$, we have the obvious holomorphic discs $\{0\} \times D^{2} \times\{z\}$. Standard methods on pseudoholomorphic curves in symplectisations [28] and the filling with holomorphic discs [13, 27] then show the following alternative: either

(i) the foliation by standard discs away from $\mathbb{R} \times B$ extends to a foliation of an embedded cylinder in $(-\infty, 0] \times Z$, or

(ii) there is a sequence of holomorphic discs whose gradient explodes.

This particular situation was investigated by Eliashberg-Hofer [15. In case (i), they show that the foliation by holomorphic discs projects to a foliation of the cylinder $\{0\} \times Z$ by embedded discs. By the definition of $J$, the Reeb orbits will be transverse to these discs, so there can be neither a periodic nor a trapped Reeb orbit (i.e. an orbit that enters the ball $B$ but does not leave it). In fact, EliashbergHofer show that $\alpha$ is then diffeomorphic to $\alpha_{\text {st }}$. In case (ii), again by the arguments of [28], one finds a periodic Reeb orbit. 
The fact that $\alpha$ is diffeomorphic to $\alpha_{\mathrm{st}}$ in the absence of periodic Reeb orbits can be interpreted as a global Darboux theorem. Another way to phrase this conclusion is as follows.

Theorem 4.1 (Eliashberg-Hofer). Let $\alpha$ be a contact form on $\mathbb{R}^{3}$ that equals the standard form $\alpha_{\text {st }}$ outside a compact set. If the Reeb vector field of $\alpha$ has a trapped orbit, then it also has a periodic orbit.

Although, in higher dimensions, a foliation by holomorphic discs is less restrictive for the Reeb dynamics, it was expected that an analogous result might hold there. This was refuted in 21, even under the additional restriction that the contact structure $\xi_{\text {st }}$ remain unchanged.

Theorem 4.2. There is a deformation of $\alpha_{\mathrm{st}}$ on $\mathbb{R}^{2 n+1}, n \geq 2$, into a contact form $\alpha=h \alpha_{\text {st }}$, with $h: \mathbb{R}^{2 n+1} \rightarrow \mathbb{R}^{+}$and $h-1$ compactly supported, such that $R_{\alpha}$ has trapped, but no periodic orbits.

The idea of the proof is to prescribe a certain dynamics, specifically, an irrational flow on a Clifford torus in $\mathbb{C}^{n} \equiv \mathbb{R}^{2 n} \times\{0\} \subset \mathbb{R}^{2 n+1}$ acting as a trap for orbits, and then to realise this dynamics as a contact Hamiltonian flow positively transverse to $\xi_{\text {st }}$ by translating these prescriptions into properties of the Hamiltonian function; the transversality condition guarantees that the Hamiltonian vector field is the Reeb vector field of a rescaled contact form. A similar dynamics for Riemannian geodesics was constructed in [5]: there are Riemannian metrics on $\mathbb{R}^{n}, n \geq 4$, equal to the Euclidean metric outside a compact set, with bounded geodesics but no periodic ones.

Eliashberg and Hofer also discuss the effect of changing the topology inside $B$ rather than just the contact form. Write $\hat{Z}$ for the cylinder with such a change of topology performed inside a ball $B \subset Z$. Here the same alternative as above holds, and the foliation by holomorphic discs in the absence of periodic Reeb orbits, i.e. alternative (i), is shown in [15] to prevent any non-trivial topology. In contrast with the previous two theorems, this Hamiltonian characterisation of the ball extends to higher dimensions. To this end, one introduces a moduli space $\mathcal{W}$ of holomorphic discs as before and studies the evaluation map $\mathcal{W} \times \mathbb{D} \rightarrow \hat{Z},((a, f), z) \mapsto f(z)$. When there are no periodic Reeb orbits, this map will be proper and surjective. By using degree-theoretic methods and the $h$-cobordism theorem, one arrives at the following result, see [23, extending [15] from three to higher dimensions. In alternative (ii), one always finds a contractible Reeb orbit whose period can be estimated by an energy integral; this accounts for the quantitative statement.

Theorem 4.3. Assume that $(M, \alpha)$ is a compact contact manifold with boundary $S^{2 n}$, such that, near the boundary, the contact form $\alpha$ looks like that of a ball $B \subset\left(Z, \alpha_{\mathrm{st}}\right)$. If $R_{\alpha}$ has no contractible periodic orbits of period smaller than $\pi$, the manifold $M$ is diffeomorphic to a ball.

For other results concerning the existence of periodic Reeb orbits on non-compact manifolds, including cotangent bundles over a non-compact base, see [8, 9, 44].

\section{Contact surgery}

We now turn our attention to closed contact manifolds $(M, \xi)$. As indicated earlier, the methods introduced by Hofer [28] — when the conditions for their applicability are satisfied - in their basic form show the existence of a contractible 
periodic Reeb orbit. This places limitations on these methods, as there are obvious examples of contact manifolds without contractible periodic Reeb orbits, as mentioned in the introduction.

Theorem 4.3 can be read as an example where $\left(\mathrm{W}^{\circ}\right)$ holds in the presence of non-trivial topology. In the same vein, one may ask if $(\mathrm{W})$ or $\left(\mathrm{W}^{\circ}\right)$ holds for any contact structure on a closed manifold of sufficiently complicated topology. One way to create non-trivial topology is by performing surgery. The most simple type of surgery is forming the connected sum $M_{1} \# M_{2}$ of two (connected) manifolds $M_{1}, M_{2}$ of the same dimension $m$ : remove an open $m$-disc from either manifold, and glue in a tube $S^{m-1} \times[-1,1]$ to connect the two manifolds. If neither manifold was a sphere, this creates non-trivial topology. Then, in particular, the belt sphere $S^{m-1} \times\{0\}$ does not bound a ball in $M_{1} \# M_{2}$.

In [28, Hofer proved $\left(\mathrm{W}^{\circ}\right)$ for closed 3 -dimensional contact manifolds $(M, \xi)$ with $\xi$ overtwisted or $M$ having non-trivial second homotopy group. According to Eliashberg's classification [14] of contact structures on the 3-sphere, all contact structures on $S^{3}$ except the standard one are overtwisted. These two results can be combined as follows.

Theorem 5.1 (Eliashberg, Hofer). Let $(M, \xi)=\left(M_{1}, \xi_{1}\right) \#\left(M_{2}, \xi_{2}\right)$ be the contact connected sum of two closed, connected contact 3-manifolds. If $(M, \xi)$ does not satisfy $\left(\mathrm{W}^{\circ}\right)$, then one of the summands $\left(M_{i}, \xi_{i}\right)$ is contactomorphic to $\left(S^{3}, \xi_{\mathrm{st}}\right)$.

In other words, $\left(\mathrm{W}^{\circ}\right)$ and a fortiori $(\mathrm{W})$ holds for all non-trivial contact connected sums. In fact, (W) is known to hold for all closed contact 3-manifolds by the work of Taubes [46], whose proof uses Seiberg-Witten theory; see [32] for an exposition of this proof.

In 24] we extended Theorem 5.1] to higher dimensions, under some topological conditions.

Theorem 5.2. Let $(M, \xi)=\left(M_{1}, \xi_{1}\right) \#\left(M_{2}, \xi_{2}\right)$ be the contact connected sum of two closed, connected contact manifolds of dimension $2 n+1 \geq 5$. Assume further that

(i) $M$ is simply connected and has torsion-free homology, or

(ii) $M$ is not simply connected.

Then, if $(M, \xi)$ does not satisfy $\left(\mathrm{W}^{\circ}\right)$, one of the summands $M_{i}$ is homeomorphic to $S^{2 n+1}$.

This theorem can potentially be used as a contact-geometric primality test for manifolds. Results of this type may even have quite concrete applications in physics. For instance, Albers et al. [3], in their study of the planar circular restricted 3-body problem, cf. 20], show that the energy hypersurface for levels slightly above the energy of the first Lagrange point is the contact connected sum of two copies of $\mathbb{R P}^{3}$. In this specific case, however, one does not need Theorem 5.1 to find a periodic orbit, for the contact form on the connected sum comes from the standard Weinstein model, so the existence of a periodic orbit is obvious and indeed known classically, cf. [2, Section 10.3], [36, Section 2], [43, §18].

The idea for proving Theorem 5.2 is to study a moduli space $\mathcal{W}$ of holomorphic discs inside the half-symplectisation $(-\infty, 0] \times M$ of $(M, \xi)$, with a Lagrangian boundary condition coming from the explicit model for the tube defining the connected sum. If $\left(\mathrm{W}^{\circ}\right)$ does not hold, one can use a deformation of the evaluation 
map $\mathcal{W} \times \mathbb{D} \rightarrow W$ to a map into $M$ to produce a filling of the belt sphere inside $M$. This filling can be shown to be a ball under either of the topological assumptions (i), (ii) in Theorem 5.2.

A different approach to statements as in that theorem has been explored in [25].

\section{OPEN BOOKS}

Besides surgery, another way to construct manifolds are so-called open books. In order to obtain a manifold $M$ of dimension $2 n+1$, we start with a compact $2 n$-dimensional manifold $\Sigma$ with non-empty boundary $\partial \Sigma$, and a diffeomorphism $\phi$ of $\Sigma$, equal to the identity near $\partial \Sigma$. The mapping cylinder

$$
V(\Sigma, \phi)=\Sigma \times[0,2 \pi] /(x, 2 \pi) \sim(\phi(x), 0)
$$

has boundary $\partial \Sigma \times S^{1}$. Form the manifold $M$ by attaching $\partial \Sigma \times D^{2}$ along the boundary:

$$
M(\Sigma, \phi)=V(\Sigma, \phi) \cup_{\partial \Sigma \times S^{1}} \partial \Sigma \times D^{2} .
$$

The submanifold $\partial \Sigma \times\{0\} \subset M(\Sigma, \phi)$ is called the binding of the open book. Its complement fibres in the obvious way over $S^{1}$ with monodromy $\phi$, and the closures of the fibres, which are copies of $\Sigma$, are called the pages. For a beautiful survey on the topology of open books see the appendix by Winkelnkemper in [41].

We assume that $\Sigma$ is oriented, the binding carries the boundary orientation of $\partial \Sigma$, and $M$ the induced orientation. A positive contact form $\alpha$ on such an open book, i.e. a 1-form satisfying $\alpha \wedge(\mathrm{d} \alpha)^{n}>0$, is said to be adapted if $\alpha$ induces a positive contact form on the binding, and $\mathrm{d} \alpha$ a positive symplectic form on the interior of each page. A contact structure is said to be supported by the open book if it can be defined by an adapted contact form.

It is not difficult to construct contact structures supported by open books, cf. 18 , Section 7.3]. One needs to start with a Liouville domain $(\Sigma, \mathrm{d} \beta)$, that is, an exact symplectic manifold such that the Liouville vector field $Y$ for $\mathrm{d} \beta$ defined by $Y\lrcorner \mathrm{d} \beta=\beta$ is transverse to $\partial \Sigma$, pointing outwards. Moreover, the monodromy diffeomorphism needs to be a symplectomorphism of $(\Sigma, \mathrm{d} \beta)$. In the sequel, it will be understood that $M(\Sigma, \phi)$ is equipped with the contact structure obtained in this way. A much deeper theorem of Giroux [26] says that in fact every contact structure on a closed manifold is supported by an open book.

In [12, $\left(\mathrm{W}^{\circ}\right)$ was proved for contact structures supported by open books under certain assumptions on the binding. Here is a simplified and more restrictive version of that result.

Theorem 6.1. If $\left(\Sigma^{2 n}, \mathrm{~d} \beta\right)$ has the structure of a subcritical Stein manifold, that is, a handle decomposition compatible with the Stein structure involving only handles up to index $n-1$, then $M(\Sigma, \phi)$ satisfies $\left(\mathrm{W}^{\circ}\right)$.

It is also possible to prove $\left(\mathrm{W}^{\circ}\right)$ for open books when suitable assumptions are made on the monodromy.

Theorem 6.2. Assume that $\phi_{1}, \ldots, \phi_{N}$ are symplectomorphisms of a Liouville manifold $(\Sigma, \mathrm{d} \beta)$ with $\phi_{1} \circ \cdots \circ \phi_{N}$ symplectically isotopic to the identity, where the diffeomorphism and isotopies are equal to the identity near the boundary. Then the contact manifold $\sqcup_{i} M\left(\Sigma, \phi_{i}\right)$ satisfies $\left(\mathrm{W}^{\circ}\right)$. 
Proof. By [4, Proposition 8.3] or [33, Theorem 1], there is a Liouville cobordism from the disjoint union

$$
\left(M_{0}, \xi_{0}\right):=\bigsqcup_{i=1}^{N} M\left(\Sigma, \phi_{i}\right)
$$

to $\left(M_{1}, \xi_{1}\right):=M\left(\Sigma, \phi_{1} \circ \cdots \circ \phi_{N}\right)$, i.e. an exact symplectic manifold $(V, \mathrm{~d} \lambda)$ with oriented boundary $\partial V=M_{1} \sqcup-M_{0}$ such that the Liouville vector field $Y$ defined by $Y\lrcorner \mathrm{d} \lambda=\lambda$ is transverse to the boundary, pointing into $V$ along $M_{0}$ (the concave end of the cobordism) and out of $V$ along $M_{1}$ (the convex end), and such that $\left.\operatorname{ker} \lambda\right|_{T M_{i}}=\xi_{i}$. By the assumption on $\phi_{1} \circ \cdots \circ \phi_{N}$ being symplectically isotopic to the identity, $\left(M_{1}, \xi_{1}\right)$ is contactomorphic to $M(\Sigma$, id $)$.

Topologically we have

$$
M(\Sigma, \text { id })=\Sigma \times S^{1} \cup_{\partial \Sigma \times S^{1}} \partial \Sigma \times D^{2}=\partial\left(\Sigma \times D^{2}\right) .
$$

The obvious Liouville structure on $\Sigma \times D^{2}$ (with corners rounded) defines a Liouville domain with boundary $M(\Sigma$, id $)$.

The symplectic completion $\hat{\Sigma}$ of the Liouville domain $(\Sigma, \mathrm{d} \beta)$ is defined by attaching a half-symplectisation to its boundary:

$$
\left(\hat{\Sigma}, \omega_{\hat{\Sigma}}\right):=(\Sigma, \mathrm{d} \beta) \cup_{\partial}\left([0, \infty), \mathrm{d}\left(\left.\mathrm{e}^{t} \beta\right|_{T(\partial \Sigma)}\right) .\right.
$$

Then the corners of $\Sigma \times D^{2}$ can be smoothed inside the symplectic manifold

$$
\left(\hat{\Sigma} \times \mathbb{C}, \omega_{\hat{\Sigma}}+\frac{r \mathrm{~d} r \wedge \mathrm{d} \theta}{\left(1+r^{2}\right)^{2}}\right),
$$

which can be partially compactified to the symplectic manifold $\hat{\Sigma} \times \mathbb{C P}^{1}$. Finally, we build the symplectic manifold

$$
\left((-\infty, 0] \times M_{0}\right) \cup_{M_{0}} V \cup_{M_{1}}\left(\left(\hat{\Sigma} \times \mathbb{C P}^{1}\right) \backslash\left(\Sigma \times D^{2}\right)\right),
$$

where the first component is again a half-symplectisation.

This manifold contains holomorphic spheres of the form $\{*\} \times \mathbb{C P}^{1}$, and one now studies the moduli space of holomorphic spheres in the homology class of these standard spheres. This approach was pioneered by McDuff 38. Compactness of this moduli space in the sense of symplectic field theory then leads to the existence of a periodic Reeb orbit in the concave end $M_{0}$. The theorem now follows directly from [22, Theorem 3.1], where $\left(\mathrm{W}^{\circ}\right)$ was proved for concave ends of a wide class of Liouville cobordisms. There, the convex end was supposed to come from a subcritical Stein manifold; the necessary modifications for the split Liouville case considered here were made in the proof of [6, Theorem 2.8].

Remark 6.3. (1) If the contact manifolds $M\left(\Sigma, \phi_{i}\right)$ are Liouville fillable for $i$ in some subset $I \subset\{1, \ldots, N\}$, that is, if there exists a Liouville cobordism from the empty set to $M\left(\Sigma, \phi_{i}\right)$ for $i \in I$, one can cap off these components of $\left(M_{0}, \xi_{0}\right)$. The preceding argument then shows that $\left(\mathrm{W}^{\circ}\right)$ holds for $\sqcup_{j \notin I} M\left(\Sigma, \phi_{j}\right)$.

(2) Now suppose that instead of having Liouville fillings we only know that the contact manifolds $M\left(\Sigma, \phi_{i}\right), i \in I$, are strongly symplectically fillable, i.e. there is a closed symplectic manifold $\left(W_{i}, \omega_{i}\right)$ with a Liouville vector field $Y$ defined near the boundary $\partial W_{i}=M_{i}$, pointing outwards and such that $\left.\operatorname{ker}(Y\lrcorner \omega_{i}\right)=\xi_{i}$. One can then still cap off these components, but now there might be symplectic spheres in these caps, so the preceding compactness argument does not go through. This problem can be circumvented with polyfolds. The symplectic manifold we 
are considering contains, in the cap at the convex end, an essential holomorphic foliation in the sense of [45. Definition 2.1], see Proposition 3.1 in that paper. As shown in [45. Corollary 1.3], the concave end $\sqcup_{j \notin I} M\left(\Sigma, \phi_{j}\right)$ then satisfies the socalled strong Weinstein conjecture: there is a null-homologous link made up of periodic Reeb orbits.

There no longer needs to be a contractible periodic Reeb orbit; see 22, Section 6.4] for an explanation of this fact. Briefly, the compactness argument allows one to find at least one finite energy plane asymptotic to a cylinder over a Reeb orbit in $(-\infty, 0] \times \sqcup_{j \notin I} M\left(\Sigma, \phi_{j}\right)$ or in the part of our symplectic manifold made up of $V$ and the caps on the $M\left(\Sigma, \phi_{i}\right), i \in I$. In the case where the caps are Liouville fillings, this latter part is still a Liouville manifold, and the theorem of Stokes prevents finite energy planes with negative punctures. Thus, a finite energy plane exists in $(-\infty, 0] \times \sqcup_{j \notin I} M\left(\Sigma, \phi_{j}\right)$, and its projection to $\sqcup_{j \notin I} M\left(\Sigma, \phi_{j}\right)$ shows that we have a contractible periodic Reeb orbit.

If the caps are not Liouville fillings, the finite energy plane might sit in the union of $V$ with the caps, which no longer gives any information about contractibility of the Reeb orbit inside $\sqcup_{j \notin I} M\left(\Sigma, \phi_{j}\right)$. Still, one finds a surface in $\sqcup_{j \notin I} M\left(\Sigma, \phi_{j}\right)$ with positive punctures asymptotic to Reeb orbits, which proves the strong Weinstein conjecture.

(3) The same argument as in (1) or (2) goes through if the $\left(M_{i}, \xi_{i}\right), i \in I$, rather than having (Liouville resp. strong symplectic) fillings, only admit co-fillings, that is, if there is a compact manifold (in the respective category) with convex boundary, where $\left(M_{i}, \xi_{i}\right)$ is one of several boundary components. Putting on these 'caps' creates new convex boundary components. These, however, do not affect the compactness argument, since holomorphic spheres cannot touch such boundaries by the maximum principle.

Here is a simple example.

Proposition 6.4. Let $\Sigma$ be a compact surface with boundary and $\phi$ a composition of left-handed Dehn twists. Then the contact manifold $M(\Sigma, \phi)$ satisfies $\left(\mathrm{W}^{\circ}\right)$. In higher dimensions, this is true for $(\Sigma, \mathrm{d} \beta)$ a Liouville manifold and $\phi$ a composition of left-handed Dehn twists along Lagrangian spheres.

Proof. When $\Sigma$ is a surface, we apply the preceding discussion to

$$
\left(M_{0}, \xi_{0}\right):=M(\Sigma, \phi) \sqcup M\left(\Sigma, \phi^{-1}\right) .
$$

By a result of Loi-Piergallini and Giroux, see [19] for an exposition, the contact manifold $M\left(\Sigma, \phi^{-1}\right)$, where the monodromy consists of right-handed Dehn twists, is Stein fillable, which means in particular Liouville fillable. Now apply Remark 6.3 (1). The higher-dimensional analogue of the result by Loi-Piergallini and Giroux is proved in [34; then the argument is completely analogous.

Remark 6.5. In the case where $M(\Sigma, \phi)$ is 3-dimensional, and if the Dehn twists on the surface $\Sigma$ making up $\phi$ are along homotopically non-trivial curves, one can argue alternatively as follows. By a result of Yllmaz [49], in this situation the contact 3 -manifold $M(\Sigma, \phi)$ is overtwisted. Then apply Hofer's result [28] mentioned in Section 5 .

One can also turn the argument on its head, as it were, and derive statements about non-existence of fillings. 
Example 6.6. Let $M(\Sigma, \phi)$ be a contact manifold whose contact structure can be defined by a contact form without contractible periodic Reeb orbits, such as that described in the introduction. Then the contact manifold $M\left(\Sigma, \phi^{-1}\right)$ is not Liouville fillable.

From the Riemann-Finsler perspective, one is of course primarily interested in proving the Weinstein conjecture for unit (co-)tangent bundles. However, we should iterate our remark from Section 3 that the principal aim in interpreting Finsler geodesic flows as Reeb flows is not to reprove theorems in Finsler geometry, but to see them as instances of results in a more general theory.

Hofer and Viterbo 29] have confirmed the Weinstein conjecture for compact connected hypersurface $M \subset T^{*} Q$ in cotangent bundles that satisfy the following conditions:

(i) $M$ is of contact type, i.e. there is a Liouville vector field $Y\left(L_{Y} \omega_{\text {can }}=\omega_{\text {can }}\right)$ defined near and transverse to $M$.

(ii) The bounded component of $T^{*} Q \backslash M$ contains the zero section $Q$.

By Section 2, this result includes the classical theorem of Lyusternik and Fet 37. on the existence of a closed geodesic on any compact Riemannian manifold.

The methods discussed in this section lead to a proof of the strong Weinstein conjecture for cotangent bundles of manifolds of the form $Q \times S^{1}$, where $Q$ is any closed manifold, see [22, Corollary 4.8].

Liouville structures on manifolds of the form $Q \times[-1,1]$, with $Q$ a compact left-quotient of one of the 3-dimensional Lie groups $\mathrm{Sol}^{3}$ or $\widetilde{\mathrm{SL}}_{2}$, were constructed in [17, 38. Then the contact manifold $M\left(Q \times[-1,1]\right.$, id), which satisfies $\left(\mathrm{W}^{\circ}\right)$ by Theorem 6.2, is diffeomorphic to $\partial\left(Q \times[-1,1] \times D^{2}\right)=Q \times S^{2}$, so at least it has the topology of the unit cotangent bundle of $Q$. However, there is no obvious identification of the contact structure on $M(Q \times[-1,1]$,id $)$ with the canonical structure on the unit cotangent bundle.

Further examples of unit cotangent bundles for which the strong Weinstein conjecture holds are given in [45, Section 3.3].

Acknowledgements. We thank Umberto Hryniewicz for his perspicacious comments on a draft version of this paper. We also thank Felix Schlenk and the referees for comments on the Finsler geometry of cotangent bundles that have resulted in the writing of Section 3 .

\section{REFERENCES}

[1] M. Abate and G. Patrizio, Finsler Metrics - A Global Approach, Lecture Notes in Mathematics 1591 (Springer-Verlag, Berlin, 1994).

[2] R. Abraham and J. E. Marsden, Foundations of Mechanics, 2nd edition (Benjamin Cummings, Reading, MA, 1978).

[3] P. Albers, U. Frauenfelder, O. van Koert and G. Paternain, Contact geometry of the restricted three-body problem, Comm. Pure Appl. Math. 65 (2012), 229-263.

[4] R. Avdek, Liouville hypersurfaces and connect sum cobordisms, arXiv:1204.3145.

[5] V. Bangert and N. Röttgen, Isoperimetric inequalities for minimal submanifolds in Riemannian manifolds: a counterexample in higher codimension, Calc. Var. Partial Differential Equations 45 (2012), 455-466.

[6] K. Barth, H. Geiges and K. Zehmisch, The diffeomorphism type of symplectic fillings, arXiv: 1607.03310.

[7] T. Barthelmé, A new Laplace operator in Finsler geometry and periodic orbits of Anosov flows, Ph.D. thesis, Strasbourg (2012); arXiv: 1204.0879. 
[8] J. B. van den Berg, F. Pasquotto and R. C. Vandervorst, Closed characteristics on non-compact hypersurfaces in $\mathbb{R}^{2 n}$, Math. Ann. 343 (2009), 247-284.

[9] J. B. van den Berg, F. Pasquotto, T. Rot and R. C. A. M. Vandervorst, On periodic orbits in cotangent bundles of non-compact manifolds, J. Symplectic Geom. 14 (2016), 11451173.

[10] H. Busemann, The Geometry of Geodesics (Academic Press, New York, 1955).

[11] S. S. Chern, W. H. Chen and K. S. Lam, Lectures on Differential Geometry, Series on University Mathematics 1 (World Scientific, River Edge, NJ, 1999).

[12] M. Dörner, H. Geiges and K. Zehmisch, Open books and the Weinstein conjecture, $Q$. J. Math. 65 (2014), 869-885.

[13] Ya. Eliashberg, Filling by holomorphic discs and its applications, Geometry of LowDimensional Manifolds, Vol. 2 (Durham, 1989), London Mathematical Society Lecture Note Series 151 (Cambridge University Press, Cambridge, 1990), 45-67.

[14] Ya. Eliashberg, Contact 3-manifolds twenty years since J. Martinet's work, Ann. Inst. Fourier (Grenoble) 42 (1992), 165-192.

[15] Ya. Eliashberg and H. Hofer, A Hamiltonian characterization of the three-ball, Differential Integral Equations 7 (1994), 1303-1324.

[16] U. Frauenfelder, C. Labrousse and F. Schlenk, Slow volume growth for Reeb flows on spherizations and contact Bott-Samelson theorems, J. Topol. Anal. 7 (2015), 407-451.

[17] H. Geiges, Examples of symplectic 4-manifolds with disconnected boundary of contact type, Bull. London Math. Soc. 27 (1995), 278-280.

[18] H. GeIges, An Introduction to Contact Topology, Cambridge Studies in Advanced Mathematics 109 (Cambridge University Press, Cambridge, 2008).

[19] H. Geiges, Contact structures and geometric topology, Global Differential Geometry, Springer Proceedings in Mathematics 17 (Springer-Verlag, Berlin, 2012), 463-489.

[20] H. Geiges, The Geometry of Celestial Mechanics, London Mathematical Society Student Texts 83 (Cambridge University Press, Cambridge, 2016).

[21] H. Geiges, N. Röttgen and K. Zehmisch, Trapped Reeb orbits do not imply periodic ones, Invent. Math. 198 (2014), 211-217.

[22] H. Geiges And K. Zehmisch, Symplectic cobordisms and the strong Weinstein conjecture, Math. Proc. Cambridge Philos. Soc. 153 (2012), 261-279.

[23] H. Geiges and K. Zehmisch, Reeb dynamics detects odd balls, Ann. Sc. Norm. Super. Pisa Cl. Sci. (5) 15 (2016), 663-681.

[24] H. Geiges and K. Zehmisch, The Weinstein conjecture for connected sums, Int. Math. Res. Not. IMRN 2016 (2016), 325-342.

[25] P. Ghiggini, K. Niederkrüger and C. Wende, Subcritical contact surgeries and the topology of symplectic fillings, J. Éc. polytech. Math. 3 (2016), 163-208.

[26] E. Giroux, Géométrie de contact: de la dimension trois vers les dimensions supérieures, Proceedings of the International Congress of Mathematicians, Vol. II (Higher Education Press, Beijing, 2002), 405-414.

[27] M. Gromov, Pseudoholomorphic curves in symplectic manifolds, Invent. Math. 82 (1985), $307-347$.

[28] H. Hofer, Pseudoholomorphic curves in symplectizations, Invent. Math. 114 (1993), 515563.

[29] H. Hofer And C. Viterbo, The Weinstein conjecture in cotangent bundles and related results, Ann. Scuola Norm. Sup. Pisa Cl. Sci. (4) 15 (1988), 411-445.

[30] U. L. Hryniewicz and P. A. S. SAlomão, Global properties of tight Reeb flows with applications to Finsler geodesic flows on $S^{2}$, Math. Proc. Cambridge Philos. Soc. 154 (2013), $1-27$.

[31] U. L. Hryniewicz and P. A. S. SAlomão, Introdução à Geometria Finsler, Publicações Matemáticas do IMPA (Rio de Janeiro, 2013).

[32] M. Hutchings, Taubes's proof of the Weinstein conjecture in dimension three, Bull. Amer. Math. Soc. (N.S.) 47 (2010), 73-125.

[33] M. KLUKAS, Open books and exact symplectic cobordisms, arXiv:1207.5647.

[34] O. VAN KOERT, Lecture notes on stabilization of contact open books, arXiv:1012.4359.

[35] P. Libermann and C.-M. Marle, Symplectic Geometry and Analytical Mechanics, Mathematics and its Applications 35 (Reidel Publishing Co., Dordrecht, 1987). 
[36] J. Llibre, R. Martínez and C. Simó, Transversality of the invariant manifolds associated to the Lyapunov family of periodic orbits near $L_{2}$ in the restricted three-body problem, $J$. Differential Equations 58 (1985), 104-156.

[37] L. A. Lyusternik And A. I. Fet, Variational problems on closed manifolds (Russian), Doklady Akad. Nauk SSSR (N.S.) 81 (1951), 17-18.

[38] D. McDuff, Symplectic manifolds with contact type boundaries, Invent. Math. 103 (1991), 651-671.

[39] H.-B. Rademacher, A sphere theorem for non-reversible Finsler metrics, Math. Ann. 328 (2004), 373-387.

[40] H.-B. Rademacher, Nonreversible Finsler metrics of positive flag curvature, A Sampler of Riemann-Finsler Geometry, Mathematical Sciences Research Institute Publications 50 (Cambridge University Press, Cambridge, 2004), 261-302.

[41] A. RANICKI, High-dimensional Knot Theory, Springer Monographs in Mathematics (SpringerVerlag, Berlin, 1998).

[42] F. SCHLEnK, Why Finsler flows naturally live in cotangent bundles, private communication, December 2016.

[43] C. L. Siegel And J. K. Moser, Lectures on Celestial Mechanics, Die Grundlehren der mathematischen Wissenschaften 187 (Springer-Verlag, Heidelberg, 1971).

[44] S. Suhr and K. Zenmisch, Linking and closed orbits, Abh. Math. Semin. Univ. Hambg. 86 (2016), 133-150.

[45] S. Suhr and K. Zehmisch, Polyfolds, cobordisms, and the strong Weinstein conjecture, Adv. Math. 305 (2017), 1250-1267.

[46] C. H. Taubes, The Seiberg-Witten equations and the Weinstein conjecture, Geom. Topol. 11 (2007), 2117-2202.

[47] A. Weinstein, On the hypotheses of Rabinowitz' periodic orbit theorems, J. Differential Equations 33 (1979), 336-352.

[48] A. Weinstein, Contact surgery and symplectic handlebodies, Hokkaido Math. J. 20 (1991), 241-251.

[49] E. Yilmaz, A note on overtwisted contact structures, Studia Sci. Math. Hungar. 48 (2011), $130-134$.

Mathematisches Institut, Universität ZU KÖln, Weyertal 86-90, 50931 Köln, GerMANY

E-mail address: mdoerner@math.uni-koeln.de

E-mail address: geiges@math.uni-koeln.de

Mathematisches Institut, WWU Münster, Einsteinstrasse 62, 48149 Münster, GerMANY

E-mail address: kai.zehmisch@uni-muenster.de 